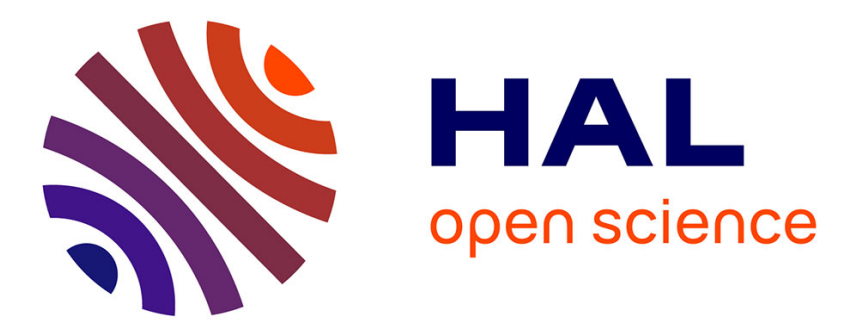

\title{
A Behavioural Dynamic Model for Constant Power Loads in Single-Phase AC Systems
}

Robert Griñó, Romeo Ortega, Emilia Fridman, Jin Zhang, Frédéric Mazenc

\section{To cite this version:}

Robert Griñó, Romeo Ortega, Emilia Fridman, Jin Zhang, Frédéric Mazenc. A Behavioural Dynamic Model for Constant Power Loads in Single-Phase AC Systems. Automatica, 2021. hal-03430910

\section{HAL Id: hal-03430910 https://inria.hal.science/hal-03430910}

Submitted on 16 Nov 2021

HAL is a multi-disciplinary open access archive for the deposit and dissemination of scientific research documents, whether they are published or not. The documents may come from teaching and research institutions in France or abroad, or from public or private research centers.
L'archive ouverte pluridisciplinaire HAL, est destinée au dépôt et à la diffusion de documents scientifiques de niveau recherche, publiés ou non, émanant des établissements d'enseignement et de recherche français ou étrangers, des laboratoires publics ou privés. 


\title{
A Behavioural Dynamic Model for Constant Power Loads in Single-Phase AC Systems *
}

\author{
Robert Griñó $^{\mathrm{a}}$, Romeo Ortega ${ }^{\mathrm{b}}$, Emilia Fridman $^{\mathrm{c}}$, Jin Zhang $^{\mathrm{c}}$, Frédéric Mazenc $^{\mathrm{d}}$ \\ ${ }^{a}$ Institute of Industrial and Control Engineering, Universitat Politécnica de Catalunya, Diagonal 647, 08028-Barcelona, Spain. \\ b ITAM, Campus Río Hondo, Río Hondo \#1, 01080-Ciudad de México, México. \\ Adaptive and Nonlinear Control Systems Lab, ITMO University, Kronverksky av., 49, 197101-Saint Petersburg, Russia.
}

${ }^{\mathrm{c}}$ Department of Electrical Engineering and Systems, Tel Aviv University, 69978-Tel Aviv, Israel.

${ }^{\mathrm{d}}$ Inria Saclay, L2S-CNRS-CentraleSupélec, 3 rue Joliot Curie, 91192, Gif-sur-Yvette, France.

\begin{abstract}
Constant power loads (CPLs) in power systems have a destabilizing effect that gives rise to significant oscillations or to network collapse, motivating the development of new methods to analyse their effect in $\mathrm{AC}$ and DC power systems. A sine qua non condition for this analysis is the availability of a suitable mathematical model for the CPL. In the case of DC systems power is simply the product of voltage and current, hence a CPL corresponds to a first-third quadrant hyperbola in the loads voltagecurrent plane. The same approach is applicable for balanced three-phase systems that, after a rotation of the coordinates to the synchronous frame, can be treated as a DC system. Modelling CPLs for single-phase (or unbalanced poly-phase) AC systems, on the other hand, is a largely unexplored area because in AC systems (active and reactive) power involves the integration in a finite window of the product of the voltage and current signals. In this paper we propose a simple dynamic model of a CPL that is suitable for the analysis of single-phase AC systems. We give conditions on the tuning gains of the model that guarantee the CPL behaviour is effectively captured.
\end{abstract}

Key words: AC electrical networks, constant power load, behavioural model, stability, delay-differential equations.

\section{Introduction}

In many electric power distribution systems and particularly in microgrids, stability problems may occur when a major proportion of the loads are electronic equipment. This kind of equipment is usually powered by cascade

\footnotetext{
* The work of Robert Griñó was supported in part by the Government of Spain through the Agencia Estatal de Investigación Project DPI2017-85404-P and in part by the Generalitat de Catalunya under Project 2017 SGR 872. The work of Emilia Fridman and Jin Zhang was supported by Israel Science Foundation (grant no. 673/19) and by the Planning and Budgeting Committee Fellowship from the Council for Higher Education, Israel. The material in this paper was not presented at any conference. Corresponding author R. Griñó.

Email addresses: roberto.grino@upc.edu (Robert Griñó), romeo.ortega@itam.mx (Romeo Ortega), emilia@tauex.tau.ac.il (Emilia Fridman), zhangjin1116@126.com (Jin Zhang), frederic.mazenc@12s. centralesupelec.fr (Frédéric Mazenc).
}

distributed architectures which are characterized by the presence of different voltage levels and power electronic converters. These converters act as interfaces between sections of different voltages in which, at last stage, loads are a combination of power electronic converters tightly regulating their output voltage, behaving as Constant Power Loads (CPLs). These architectures are common in information and communication technology facilities where the many telecom switches, wireless communication base stations, and data center servers act as CPLs. It is well-known that CPLs introduce a destabilizing effect that gives rise to significant oscillations or even voltage collapse, and hence they are considered to be the most challenging component of the standard load modelreferred to as the ZIP model in power system stability analysis. See (Molinas, Moltoni, Fascendini, Suul \& Undeland 2008, Karimipour \& Salmasi 2015, Machado 2019, Singh, Gautam \& Fulwani 2017) for a recent review of the literature and (Matveev, Machado, Ortega, Schiffer \& Pyrkin 2020) for a detailed analysis of the effect of CPLs on the power systems behavior. 
The growing presence of CPLs in modern installations significantly aggravates this issue, hence motivating the development of new methods to analyze their effect in $\mathrm{AC}$ and DC power systems. To carry out this analysis it is necessary to dispose of a mathematical model that suitably describes the behaviour of the CPL. In the case of a DC system a suitable model for the CPL is simply a firstthird quadrant hyperbola in the loads voltage-current plane, $i(t)=\frac{P}{v(t)}$, and, consequently, the load incrementally behaves as a negative resistance contributing to reduce the relative stability of the electrical network and even to destabilize it (Marx, Magne, Nahid-Mobarakeh, Pierfederici \& Davat 2012, Riccobono \& Santi 2014). The same model is applicable for balanced three-phase systems that, after a rotation of the coordinates to the synchronous frame, can be treated as a DC system. Modeling CPLs for single-phase (or unbalanced multi-phase) AC systems, on the other hand, is a largely unexplored area because of the definition of active power $(P(t))$ in AC systems (IEEE 2010, Garcia-Canseco, Griñó, Ortega, Salichs \& Stankovic 2007), which involves the integration in a finite moving window of the product of the voltage and current signals, more precisely, for a singlephase $\mathrm{AC}$ system $^{1}$ we have

$$
P(t)=\frac{1}{T} \int_{t-T}^{t} v(s) i(s) d s,
$$

where $v(t)$ is the voltage and $i(t)$ the current. Throughout the paper we make the following assumption.

Assumption 1 The voltage $v(t)$ is T-periodic, i.e., $v(t)=v(t+T)$, for all $t \geq 0$.

In this paper we propose a simple dynamic model of a CPL that is suitable for the analysis of single-phase AC systems $^{2}$. Because of space limitations we have concentrated in the emulation of active power. However, the extension to include reactive power is immediate and is explained in Section 6. To derive the model we adopt a control theory perspective and reformulate the problem as the design of a negative feedback controller wrapped around the output of a dynamical system that computes $P(t)$. The voltage, required in the computation of $P(t)$, is viewed as an external periodic signal. The control objectives are to drive $P(t)$ to some constant desired value and to ensure that the current is in phase with the voltage. In spite of the apparent simplicity of the aforementioned control problem, it is a far from trivial task because the dynamics of the power computation model

\footnotetext{
1 It is worth to remark that this definition computes the active power $(\mathrm{W})$ for sinusoidal and nonsinusoidal (general $T$-periodic) AC single-phase systems and it is the causal version of the expression that appears in (IEEE 2010).

2 For AC poly-phase systems definition (1) changes to $P(t)=\frac{1}{T} \int_{t-T}^{t} \mathbf{v}^{\top}(s) \mathbf{i}(s) d s$ where $\mathbf{v}(t)$ and $\mathbf{i}(t)$ are, for example, the line-to-neutral voltages vector and the line currents vector, respectively.
}

is a ( $T$-periodic) linear time-varying delay-differential equation (LTV-DDE), complicating the design of the required controller.

We consider in the paper two controllers: a simple proportional one with a constant bias input and a classical proportional-integral (PI) controller. A detailed analysis of the stability of the closed-loop is carried out using advanced techniques of delay-differential systems. Thus, the stability analysis under PI controller employs a recent time-delay approach to averaging (Fridman \& Zhang 2020). The outcome of this study is the definition of regions in the space of the controller parameters for which asymptotic stability is ensured. To check how conservative the bounds derived from the theoretical analysis are, we carry out extensive numerical simulations and the computation of the more relevant characteristic multipliers of the linear periodic DDEs that arise.

To the best of the authors' knowledge this is the first attempt to develop a mathematically well-founded model for AC CPL's. Most of the results been restricted to numerical derivations (Y. Dong, W. Liu, Z. Gao \& X. Zhang 2008) or invoking various kinds of approximations to the actual phenomenon (Molinas et al. 2008).

\section{Problem Formulation}

In this section we describe the dynamical system that computes the active power $P(t)$ defined in (1) as an LTV operator $\Sigma:(v, i) \mapsto P$. From (1) we get the DDE satisfied by $P(t)$ as

$$
\dot{P}(t)=\frac{1}{T}[p(t)-p(t-T)] .
$$

where, for ease of reference, we have defined the instantaneous active power

$$
p(t)=v(t) i(t)
$$

A block diagram description of the system $\Sigma$ is given in the green box of Fig. 1, where $v(t)$ is a fixed $T$-periodic, external signal and $i(t)$ as the "control" to be defined. As explained in the introduction, to derive our dynamic model of the AC CPL we adopt a control theory perspective. To generate the input signal $i(t)$ of the system $\Sigma$ we propose a classical output feedback configuration with $\Sigma_{c}: \tilde{P} \mapsto u$ the controller to be defined, and $\tilde{P}(t):=P_{\star}-P(t)$, with $P_{\star}$ the desired value for $P(t)$. To capture our objective of ensuring that, in steady-state, the voltage and the current are in phase, we define the latter as

$$
i(t)=u(t) v(t)
$$

In this case, the "in-phase" requirement translates to the condition that $u(t)$ converges to a constant value. A 


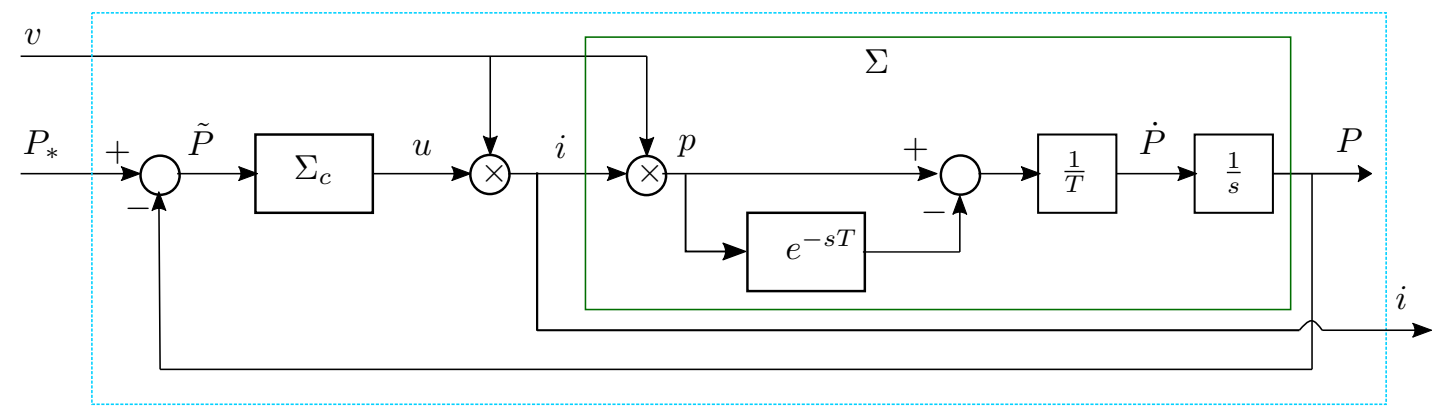

Fig. 1. Block diagram representation of the system (6) in closed-loop with feedback controller $\Sigma_{c}$. The light blue box encloses all the CPL device with port voltage and desired active power inputs and current output.

block diagram description of the overall system is given in Fig. 1.

To complete the description of the mathematical model of the system $\Sigma$, we note that from (3) and (4) we have $p(t)=v^{2}(t) u(t)$. Then (1) can be rewritten as

$$
P(t)=\frac{1}{T} \int_{t-T}^{t} v^{2}(\ell) u(\ell) d \ell
$$

revealing that $P(t)$ is just an average of $v^{2}(t) u(t)$. Differentiating the latter equation, we arrive at the final description of our system

$$
\dot{P}(t)=\frac{v^{2}(t)}{T}[u(t)-u(t-T)] .
$$

We are now in position to formulate our controller design problem.

Control problem formulation Consider the LTV, delay-differential, periodic, scalar system (6). Given $P_{\star}>0$, design a controller $\Sigma_{c}: \tilde{P} \rightarrow u$ such that

$$
\begin{aligned}
& \lim _{t \rightarrow \infty} P(t)=P_{\star} \\
& \lim _{t \rightarrow \infty} u(t)=u_{\star}
\end{aligned}
$$

where $u_{\star}>0$ is an arbitrary constant.

In the following two sections we will present two controllers $\Sigma_{c}$ : a simple proportional one with a constant bias input and a classical proportional-integral (PI) controller. For both schemes, we give conditions on their tuning gains that ensure the control objective (7) is satisfied with all signals remaining bounded.

Remark 1 A particular case of practical interest is

$$
v(t)=\sqrt{2} V \sin \left(\frac{2 \pi}{T} t\right)
$$

where $V>0$ is the RMS value of the voltage $v(t)$ defined as

$$
V^{2}:=\frac{1}{T} \int_{0}^{T} v^{2}(\ell) d \ell
$$

\section{A Simple Proportional plus Constant Bias Controller}

The main result of this section is given in the proposition below whose proof, to enhance readability, is given in Appendix A.

Proposition 1 Consider the simple proportional plus constant bias control law

$$
u(t)=k_{p} \tilde{P}(t)+u_{\star}
$$

with $u_{\star}=\frac{1}{V^{2}} P_{\star}$ and $V$ the $R M S$ value of the voltage $v(t)$ given by (9), which satisfies Assumption 1. For all values of $k_{p}$ in the interval $\left(0, \frac{1}{V}\right)$ the conditions (7) are satisfied-with the convergence being exponential. $\square \square \square$

Remark 2 It is shown in the proof that, when $P_{\star}$ is fixed, the $u_{\star}$ given in Proposition 1 is the unique value that can be obtained for the limit of $u(t)$ if both $P(t)$ and $u(t)$ converge to constant values. From the proof it also follows that $\frac{\ln k_{p} V}{T}$ is an upperbound on the exponential rate of convergence.

Remark 3 As it is well-known, to enhance the robustness of the system, it is necessary to implement feedback - as opposed to open-loop - control laws. In any case, it is of mathematical interest to observe from (A.1) that setting $k_{p}=0$, that is, considering the feedforward controller $u=u_{\star}$, convergence of the error to zero is achieved in finite time. Actually, it is also possible to show that convergence is preserved even for negative values of $k_{p}$ in the interval $\left(-\frac{1}{V}, \frac{1}{V}\right)$ and that the trajectories of the closed-loop system remain bounded for all $k_{p}$. Since the analysis is quite involved, and the boundedness property not very informative, it is omitted for brevity. 


\section{A Proportional-Integral Controller}

In this section we analyze the behavior of the closed-loop system when $\Sigma_{c}$ is a PI controller. That is,

$$
\begin{aligned}
\dot{x}_{c}(t) & =\tilde{P}(t) \\
u(t) & =k_{p} \tilde{P}(t)+k_{i} x_{c}(t)
\end{aligned}
$$

with $k_{p} \geq 0$ and $k_{i}>0$. As in the previous controller our objective is to find conditions on $\left(k_{p}, k_{i}\right)$ - given in terms of feasibility of linear matrix inequalities (LMIs) - such that the control objective (7) is satisfied. We consider two possible scenarios, when $v^{2}(t)$ is $T$-periodic, or $\frac{T}{2}$ periodic. The motivation to consider the two cases is two-fold: on one hand, the LMIs are less conservative when considering smaller periods. On the other hand, the case of practical interest ( 8$)$ is ${ }^{3}$, indeed, $\frac{T}{2}$-periodic.

The proposition below, whose proof is given in Appendix $\mathrm{B}$, pertains to the $T$-periodic case.

Proposition 2 Let $v^{2}(t)$ be $T$-periodic.

(i) Consider the PI controller given by (11) with $k_{p}>0$ and $k_{i}>0$. Given $k_{p}^{M}>0$ and $k_{i}^{M}>0$, if there exist scalars $r>0, r_{1}>0, s>0$ and $q>0$ such that the following LMIs are satisfied

$$
\Xi_{0}=-s+2 v_{M}^{4}\left[\left(k_{p}^{M}\right)^{2} s+\left(k_{i}^{M}\right)^{2}(r+q)\right]+\frac{1}{T^{2}} r_{1}<0,
$$

and

$\Xi\left(k_{p}^{M}, k_{i}^{M}\right)=\left[\begin{array}{cccc}\Xi_{11}\left(k_{p}^{M}, k_{i}^{M}\right) & V^{2} & 1 & 1 \\ * & -\frac{4}{T} r & -1 & -1 \\ * & * & -\frac{1}{v_{M}^{4} T} & 0 \\ * & * & * & -\frac{1}{v_{M}^{4} T} r_{1}\end{array}\right]<0$,

where we defined the function

$\Xi_{11}\left(k_{p}^{M}, k_{i}^{M}\right):=-2 V^{2}+2 v_{M}^{4} T\left[\left(k_{p}^{M}\right)^{2} s+\left(k_{i}^{M}\right)^{2}(r+q)\right]$,

and

$$
v_{M}:=\max _{t \in[0, T]} v(t),
$$

then (7) is satisfied for all $k_{p} \in\left[-k_{p}^{M}, k_{p}^{M}\right]$ and $k_{i} \in$ $\left(0, k_{i}^{M}\right]$.

(ii) Consider the I controller given by (11) with $k_{p}=0$ and $k_{i}>0$. Given $k_{i}^{M}>0$, if there exist scalars $r>0$

\footnotetext{
3 Another real case of interest is when $v(t)$ is a $T$-periodic signal with only odd harmonics (with zero average). In this situation $v^{2}(t)$ is also a $\frac{T}{2}$-periodic signal.
}

and $q>0$ such that the following $L M I$

$$
\left[\begin{array}{ccc}
-2 V^{2}+v_{M}^{4} T\left(k_{i}^{M}\right)^{2}(r+q) & V^{2} & 1 \\
* & -\frac{4}{T} r & -1 \\
* & * & -\frac{1}{v_{M}^{4} T} q
\end{array}\right]<0
$$

holds, then (7) is satisfied for all $k_{i} \in\left(0, k_{i}^{M}\right]$.

As indicated above, if $v^{2}(t)$ has a smaller fundamental period $\frac{T}{2}$, a less conservative LMI condition may be derived. This result is contained in the corollary below, whose proof is given in Appendix C.

Corollary 1 Let $v^{2}(t)$ be $\frac{T}{2}$-periodic.

(i) Consider the PI controller given by (11) with $k_{p}>0$ and $k_{i}>0$. Given $k_{p}^{M}>0$ and $k_{i}^{M}>0$, if there exist scalars $r>0, r_{1}>0, s>0$ and $q>0$ such that the following LMIs are satisfied

$$
\hat{\Xi}_{0}=-s+2 v_{M}^{4}\left[\left(k_{p}^{M}\right)^{2} s+\left(k_{i}^{M}\right)^{2}\left(\frac{r}{2}+q\right)\right]+\frac{1}{T^{2}} r_{1}<0,
$$

and

$\hat{\Xi}\left(k_{p}^{M}, k_{i}^{M}\right)=\left[\begin{array}{cccc}\hat{\Xi}_{11}\left(k_{p}^{M}, k_{i}^{M}\right) & V^{2} & 1 & 1 \\ * & -\frac{8}{T} r & -1 & -1 \\ * & * & -\frac{16}{9 v_{M}^{4} T} & 0 \\ * & * & * & -\frac{1}{v_{M}^{4} T} r_{1}\end{array}\right]<0$,

where we defined the function

$\hat{\Xi}_{11}\left(k_{p}^{M}, k_{i}^{M}\right):=-2 V^{2}+2 v_{M}^{4} T\left[\left(k_{p}^{M}\right)^{2} s+\left(k_{i}^{M}\right)^{2}\left(\frac{r}{2}+q\right)\right]$,

and $v_{M}$ is given by (14), then (7) is satisfied for all $k_{p} \in$ $\left[-k_{p}^{M}, k_{p}^{M}\right]$ and $k_{i} \in\left(0, k_{i}^{M}\right]$.

(ii) Consider the I controller given by (11) with $k_{p}=0$ and $k_{i}>0$. Given $k_{i}^{M}>0$, if there exist scalars $r>0$ and $q>0$ such that the following $L M I$

$$
\left[\begin{array}{ccc}
-2 V^{2}+v_{M}^{4} T\left(k_{i}^{M}\right)^{2}\left(\frac{r}{2}+q\right) & V^{2} & 1 \\
* & -\frac{8}{T} r & -1 \\
* & * & -\frac{16}{9 v_{M}^{4} T} q
\end{array}\right]<0
$$

holds, then (7) is satisfied for all $k_{i} \in\left(0, k_{i}^{M}\right]$.

Remark 4 As it is seen from LMIs of Proposition 2 and Corollary 1 , given $T>0$, these LMIs hold with $k_{p}^{M}=k_{i}^{M}=0$. Thus, these LMIs hold for sufficiently small PI gains, i.e. (7) is always guaranteed for small enough $k_{p} \geq 0$ and $k_{i}>0$. 
Remark 5 The claim that LMIs (16) and (17) are less conservative than the LMIs (12) and (13) can be established noting that $\hat{\Xi}_{0}-\Xi_{0} \leq 0$ and $\hat{\Xi}(\cdot)-\Xi(\cdot) \leq 0$ hold. Note also that LMI (13) with $k_{p}=0$ is more conservative than LMI (15) in Proposition 2 because its 11term $-2 V^{2}+2 v_{M}^{4} T\left(k_{i}^{M}\right)^{2}(r+q)$ is larger than 11-term $-2 V^{2}+v_{M}^{4} T\left(k_{i}^{M}\right)^{2}(r+q)$ in (15). The same holds for LMIs (17) and (18) in Corollary 1.

\section{Numerical Simulation Results}

This section shows some simulation results and numerical stability assessment, using the characteristic multipliers of the monodromy matrix of the closed-loop delaydifferential system, for the AC sinusoidal voltage (8) with $V=230.0 \mathrm{~V}, T=0.02 \mathrm{~s}$. The computation of the characteristic multipliers has been done with the algorithm eigTMN (Breda, Maset \& Vermiglio 2015).

Remark 6 The proposed behavioural model has been extensively tested numerically against step variations of the RMS value of the grid voltage, $V$, and there are no stability problems for reasonable variations of that value around the nominal one. Besides, the main theoretical result in Proposition 2 can be extended to a variable in time $V \geq V_{0}>0$ that takes values in the given interval. This extension is not in the scope of the current paper.

\section{$5.1 P$ controller}

Using the controller in eq. (10), the closed-loop dynamics is

$$
\dot{P}(t)=\frac{-k_{p}}{T} v^{2}(t) P(t)+\frac{k_{p}}{T} v^{2}(t) P(t-T)
$$

and the sufficient condition in Proposition 1 states that it would be stable for $k_{p} \in\left(0, \frac{1}{V}\right)=\left(0,4.348 \cdot 10^{-3}\right)$.

The numerical study of the stability of eq. (19) is shown in Fig. 2 where the maximum absolute value characteristic multiplier is plotted against $k_{p}$. As it can be observed, the closed-loop system is stable for all positive values of $k_{p}$-revealing that, at least for this particular numerical scenario, the theoretical analysis is very conservative. As it can be seen from the figure, the closedloop system tends to be marginally stable as $k_{p} \rightarrow \infty$.

Fig. 3 shows the power, $P(t)$, and the control signal, $u(t)$, time responses for a step reference in power, $P_{\star}=$ $1000 \mathrm{~W}$, for three different values of $k_{p}$. As it can be observed, the steady-state error is zero and the overshoot in the transient increases as $k_{p}$ gets bigger. In Fig. 4 we plot the voltage applied to the CPL and the computed current for the same three different values of $k_{p}$. After the transient, with bigger overshoot as $k_{p}$ grows, the current is sinusoidal and perfectly in-phase with the voltage.

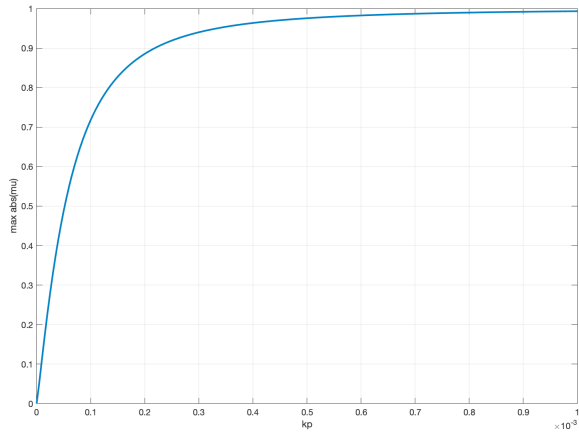

Fig. 2. Maximum absolute value characteristic multiplier vs. $k_{p}$.


Fig. 3. $P_{\star}$ and $P(t)$ (left), and $u(t)$ (right) for the proportional controller.

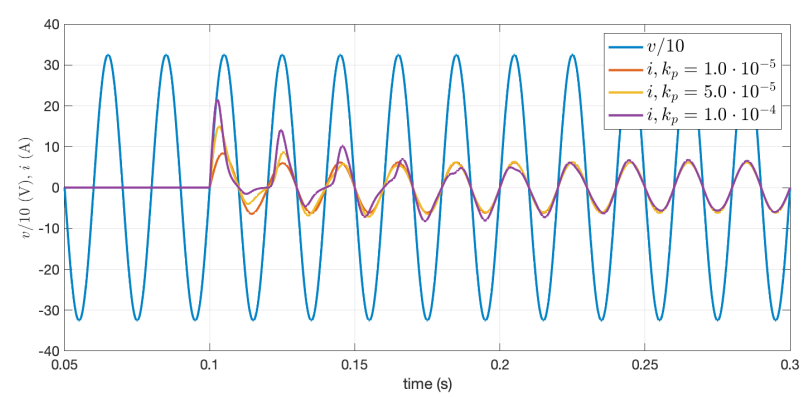

Fig. 4. Voltage, $v(t) / 10$, and CPL current $i(t)$ for the proportional controller.

\subsection{PI controller}

In this subsection we investigate numerically the PI controller (11) with $k_{p}>0, k_{i}>0$, which results in the closed-loop dynamics

$$
\begin{aligned}
{\left[\begin{array}{c}
\dot{\tilde{P}}(t) \\
\dot{x}_{c}(t)
\end{array}\right]=} & {\left[\begin{array}{cc}
\frac{-k_{p}}{T} v^{2}(t) & \frac{-k_{i}}{T} v^{2}(t) \\
1 & 0
\end{array}\right]\left[\begin{array}{l}
\tilde{P}(t) \\
x_{c}(t)
\end{array}\right] } \\
& +\left[\begin{array}{cc}
\frac{k_{p}}{T} v^{2}(t) & \frac{k_{i}}{T} v^{2}(t) \\
0 & 0
\end{array}\right]\left[\begin{array}{l}
\tilde{P}(t-T) \\
x_{c}(t-T)
\end{array}\right]
\end{aligned}
$$

Fig. 5 (left) shows the stability chart in the parameter plane $\left(k_{p}, k_{i}\right)$, where the shaded area in solid grey corresponds to unstable behaviour. This information has been obtained looking for the maximum absolute value 

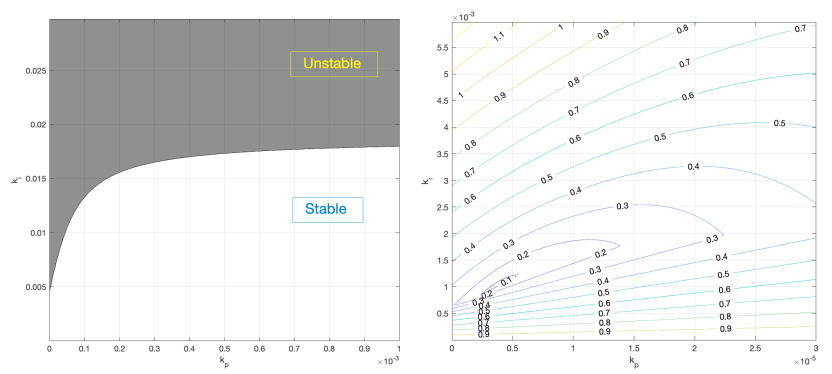

Fig. 5. $\left(k_{p}, k_{i}\right)$ stability charts: (left) stability regions and (right) contour lines for the maximum absolute value characteristic multiplier near $k_{p}=0$ and $k_{i}=0$.

characteristic multiplier of a rectangular sampling of the parameter space $k_{p}-k_{i}$. In Fig. 5 (right) we show the contour lines for the maximum absolute value characteristic multiplier in the plane $\left(k_{p}, k_{i}\right)$ for $k_{p}$ and $k_{i}$ close to zero. It is worth to mention that in the other three quadrants of the plane $k_{p}-k_{i}$ the system is unstable.


Fig. 6. $P_{\star}$ and $P(t)$ (left) and $u(t)$ (right) for the PI controller.

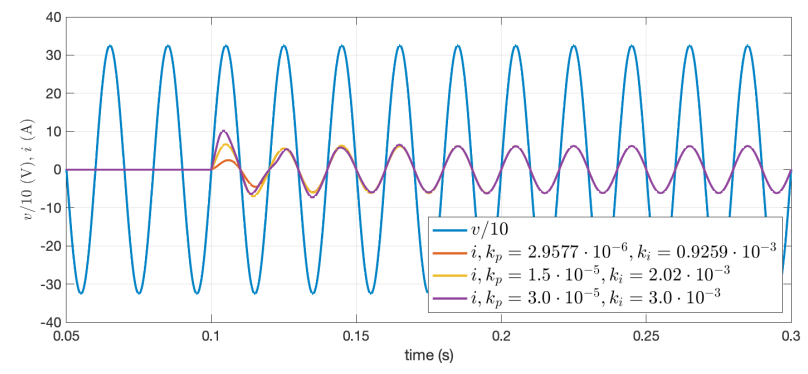

Fig. 7. Voltage, $v(t) / 10$ and CPL current $i(t)$ for the PI controller.

Looking for the $k_{p}$ and $k_{i}$ values that give the minimum maximum absolute value characteristic multiplier $\left(\min _{k_{p}, k_{i}} \max _{l}\left|\mu_{l}\left(k_{p}, k_{i}\right)\right|\right)$ results ${ }^{4}$ in $k_{p}=2.95767$. $10^{-6}, k_{i}=0.92594 \cdot 10^{-3}$ with a maximum absolute value characteristic multiplier, $\max _{l}\left|\mu_{l}\right|=0.048368$.

Fig. 6 shows responses of the power, $P(t)$, and the control signal, $u(t)$, to a step reference in power, $P_{\star}=1000$

\footnotetext{
4 These optimum has been found using several tries of a Nelder-Mead optimization algorithm. So, it can not be assured that it is the global minimum but it is the best minimum found.
}

$\mathrm{W}$, for different values of $k_{p}$ and $k_{i}$. As it can be observed, the steady-state error is zero due to the integrator included in the controller. The red curve in the left plot and the blue curve in the right plot correspond to the $k_{p}$ and $k_{i}$ values of the minimum maximum absolute value characteristic multiplier commented in the previous paragraph. As seen from these plots the resulting transient performance is excellent with no overshoot. In Fig. 7 appears the (scaled) voltage $(v / 10)$ applied to the $\mathrm{CPL}$ and the computed current for the same three different values of $k_{p}$ and $k_{i}$. After the transient, the currents are sinusoidal and perfectly in-phase with the voltage. Table 1 below summarizes the numerical LMI results with $v_{M}=\sqrt{2} V$ running the solver with different $k_{p}^{M}$.

\section{Concluding Remarks and Future Research}

We have presented in the paper a possible scenario for the emulation of CPLs in single-phase AC systems. The inputs of the system are the voltage signal, which is only assumed to be periodic, and the desired value of the constant power load. The in-phase current that will generate this active power is calculated with a dynamic controller. We have tried three different versions of the latter: proportional+bias, integral and proportional+integral, for which we dispose of a rigorous theoretical analysis to determine bounds on their tuning gains, hence these results are compared with numerical validation.

Because of space limitations, we have concentrated in the emulation of active power. However, the extension to include reactive power is immediate. Indeed, reactive power can be calculated ${ }^{5}$ as $Q(t)=\frac{-1}{2 \pi} \int_{t-T}^{t} \dot{v}(s) i(s) d s$, see (Garcia-Canseco et al. 2007) for a control-oriented discussion on reactive power. Taking into account this definition, a single-phase constant reactive power load can be emulated changing, in Fig. 1, the voltage carrier, $v(t)$, by its derivative, $\dot{v}(t)$. Obviously, it is also possible to emulate a constant active and reactive power load using two closed-loop systems: one to compute the current for the constant active power load and the other to compute the current for the constant reactive power load. Then, the total current is the sum of the currents from each system as they are in quadrature.

\section{References}

Breda, D., Maset, S. \& Vermiglio, R. (2015), Stability of Linear Delay-differential Equations - A Numerical Approach with MATLAB, Springer.

Fridman, E. (2014), Introduction to Time-delay Systems: Analysis and Control, Birkhauser.

\footnotetext{
5 This definition characterizes the reactive power of a load that can be fully compensated using a shunt capacitor or a shunt inductor at its port variables and, for $\mathrm{AC}$ sinusoidal systems, single-phase and poly-phase, coincides with the usual equations to compute the reactive power.
} 
Table 1

Maximum value of $k_{i}^{M}$ for different $k_{p}^{M}$ via LMIs of Proposition 2 and Corollary 1.

\begin{tabular}{l|lllll}
\hline$k_{p}^{M}$ & 0 & $1 \cdot 10^{-6}$ & $2 \cdot 10^{-6}$ & $4 \cdot 10^{-6}$ & $6 \cdot 10^{-6}$ \\
\hline Proposition 2 & $2.2923 \cdot 10^{-4}$ & $1.6027 \cdot 10^{-4}$ & $1.5466 \cdot 10^{-4}$ & $1.2985 \cdot 10^{-4}$ & $0.7140 \cdot 10^{-4}$ \\
Corollary 1 & $3.1077 \cdot 10^{-4}$ & $2.1727 \cdot 10^{-4}$ & $2.0967 \cdot 10^{-4}$ & $1.7604 \cdot 10^{-4}$ & $0.9680 \cdot 10^{-4}$ \\
\hline
\end{tabular}

Fridman, E. \& Shaikhet, L. (2016), 'Delay-induced stability of vector second-order systems via simple Lyapunov functionals', Automatica 74, 288-296.

Fridman, E. \& Zhang, J. (2020), 'Averaging of linear systems with almost periodic coefficients: A time-delay approach', Automatica 122, 109287.

Garcia-Canseco, E., Griñó, R., Ortega, R., Salichs, M. \& Stankovic, A. (2007), 'Power factor compensation of electrical circuits: The nonlinear non-sinusoidal case', IEEE Control Systems Magazine 27(4), 46-59.

IEEE (2010), 'IEEE standard definitions for the measurement of electric power quantities under sinusoidal, nonsinusoidal, balanced, or unbalanced conditions, IEEE Std 1459-2010'.

Karimipour, D. \& Salmasi, F. (2015), 'Stability analysis of ac microgrids with constant power loads based on popov's absolute stability criterion', IEEE Trans. on Circuits and Systems II: Express Briefs 62(7), 696-700.

Machado, J. (2019), Some Problems on the Analysis and Control of Electrical Networks with Constant Power Loads, PhD Thesis, Universite Paris-Saclay.

Marx, D., Magne, P., Nahid-Mobarakeh, B., Pierfederici, S. \& Davat, B. (2012), 'Large signal stability analysis tools in dc power systems with constant power loads and variable power loads - a review', IEEE Trans. on Power Electronics 27(4), 1773-1787.

Matveev, A. S., Machado, J. E., Ortega, R., Schiffer, J. \& Pyrkin, A. (2020), 'A tool for analysis of existence of equilibria and voltage stability in power systems with constant power loads', IEEE Trans. on Automatic Control 65(11), 4726-4740.

Mazenc, F., Malisoff, M. \& Niculescu, S. (2017), 'Stability and control design for time-varying systems with time-varying delays using a trajectory-based approach', SIAM J. Control Optim. 55(1), 533-556.

Molinas, M., Moltoni, D., Fascendini, G., Suul, J. \& Undeland, T. (2008), Constant power loads in ac distribution systems: An investigation of stability, in '2008 IEEE International Symposium on Industrial Electronics', pp. 1531-1536.

Riccobono, A. \& Santi, E. (2014), 'Comprehensive review of stability criteria for dc power distribution systems', IEEE Trans. on Industry Applications 50(5), 3525-3535.

Singh, S., Gautam, A. \& Fulwani, D. (2017), 'Constant power loads and their effects in dc distributed power systems: A review', Renewable and Sustainable Energy Revs. 72, 407421.

Solomon, O. \& Fridman, E. (2013), 'New stability conditions for systems with distributed delays', Automatica 49(11), 34673475 .

Y. Dong, W. Liu, Z. Gao \& X. Zhang (2008), Study of a simulation model of ac constant power load, in 'TENCON 2008 - 2008 IEEE Region 10 Conference'.

\section{A Proof of Proposition 1}

Substituting $u(t)=k_{p}\left[P_{\star}-P(t)\right]+\frac{1}{V^{2}} P_{\star}$ into (5) we obtain

$$
P(t)=\frac{k_{p}}{T} \int_{t-T}^{t} v^{2}(\ell)\left[P_{\star}-P(\ell)\right] d \ell+P_{\star},
$$

or, equivalently,

$$
\tilde{P}(t)=-\frac{k_{p}}{T} \int_{t-T}^{t} v^{2}(\ell) \tilde{P}(\ell) d \ell
$$

Consequently, taking into account that the function $v(t)$ is $T$-periodic, we obtain

$$
\begin{aligned}
|\tilde{P}(t)| & \leq \frac{k_{p}}{T} \int_{t-T}^{t} v^{2}(\ell)|\tilde{P}(\ell)| d \ell \\
& \leq \frac{k_{p}}{T} \int_{0}^{T} v^{2}(\ell) d \ell \sup _{\ell \in[t-T, t]}|\tilde{P}(\ell)| \\
& =k_{p} V \sup _{\ell \in[t-T, t]}|\tilde{P}(\ell)|, \quad t \geq 0 .
\end{aligned}
$$

By using Lemma 1 in (Mazenc, Malisoff \& Niculescu $2017)$ with the condition $k_{p} V<1$, we arrive at $|\tilde{P}(t)| \leq$ $\sup _{\ell \in[-T, 0]}|\tilde{P}(\ell)| e^{\frac{\ln \left(k_{p} V\right)}{T} t}$. Thus, (7) holds.

\section{B Proof of Proposition 2}

We will employ Lemma 1 given in Appendix D.

(i) To complete the proof of (i) we make the following simple, but important, observation. For the PI control law (11), we have the relation

$$
\begin{aligned}
& u(t)-u(t-T)=\int_{t-T}^{t} \dot{u}(\xi) d \xi \\
& =k_{p} \int_{t-T}^{t} \dot{\tilde{P}}(\xi) d \xi+k_{i} \int_{t-T}^{t} \tilde{P}(\xi) d \xi
\end{aligned}
$$

Substituting (B.1) into (6) and taking into account $\dot{\tilde{P}}(t)=-\dot{P}(t)$, we arrive at

$$
\dot{\tilde{P}}(t)=-\frac{k_{p}}{T} v^{2}(t) \int_{t-T}^{t} \dot{\tilde{P}}(\xi) d \xi-\frac{k_{i}}{T} v^{2}(t) \int_{t-T}^{t} \tilde{P}(\xi) d \xi
$$

Then, conditions (7) are satisfied if (B.2) is asymptotically stable.

For the stability analysis of the time-varying system (B.2) with the $T$-periodic coefficient $v^{2}(t)$, we suggest to 
use the averaging method. We will employ a constructive time-delay approach to averaging introduced recently in (Fridman \& Zhang 2020). Following this approach, we will integrate (B.2) on $t \in[t-T, t]$ for $t \geq T$. Note that similar to (Fridman \& Shaikhet 2016), we get

$$
\frac{1}{T} \int_{t-T}^{t} \dot{\tilde{P}}(\xi) d \xi=\frac{1}{T}[\tilde{P}(t)-\tilde{P}(t-T)]=\frac{d}{d t}[\tilde{P}(t)-G],
$$

where

$$
G=\frac{1}{T} \int_{t-T}^{t}(\xi-t+T) \dot{\tilde{P}}(\xi) d \xi .
$$

Denote $z(t):=\tilde{P}(t)-G$. Then, integrating (B.2) and taking into account (B.3) we arrive at

$$
\begin{aligned}
\dot{z}(t)= & -\frac{k_{p}}{T^{2}} \int_{t-T}^{t} v^{2}(\xi) \int_{\xi-T}^{\xi} \dot{\tilde{P}}(\theta) d \theta d \xi \\
& -\frac{k_{i}}{T^{2}} \int_{t-T}^{t} v^{2}(\xi) \int_{\xi-T}^{\xi} \tilde{P}(\theta) d \theta d \xi \\
= & -\frac{k_{p}}{T^{2}} \int_{t-T}^{t} v^{2}(\xi) \int_{\xi-T}^{\xi} \tilde{\tilde{P}}(\theta) d \theta d \xi \\
& -\frac{k_{i}}{T^{2}} \int_{t-T}^{t} v^{2}(\xi) \int_{\xi-T}^{\xi} d \theta d \xi \tilde{P}(t) \\
& +\frac{k_{i}}{T^{2}} \int_{t-T}^{t} v^{2}(\xi) \int_{\xi-T}^{\xi}[\tilde{P}(t)-\tilde{P}(\theta)] d \theta d \xi, \quad t \geq T .
\end{aligned}
$$

The latter equation can be written as

$$
\dot{z}(t)=-k_{i} V^{2} \tilde{P}(t)+k_{i} Y+k_{i} X, \quad t \geq T
$$

where

$$
\begin{aligned}
& Y=\frac{1}{T^{2}} \int_{t-T}^{t} \int_{\xi-T}^{\xi} \int_{\theta}^{t} v^{2}(\xi) \dot{\tilde{P}}(\tau) d \tau d \theta d \xi \\
& X=-\frac{k_{p}}{k_{i} T^{2}} \int_{t-T}^{t} \int_{\xi-T}^{\xi} v^{2}(\xi) \dot{\tilde{P}}(\theta) d \theta d \xi
\end{aligned}
$$

Summarizing, if $\tilde{P}(t)$ is a solution to (B.2), then it satisfies the time-delay system (B.5). Therefore, the stability of the time-delay system guarantees the stability of the original system.

We will derive the stability conditions for the time-delay system (B.5) via Lyapunov-Krasovskii's method. Towards this end, we choose the function

$$
V_{z}=z^{2}(t)
$$

Differentiating $V_{z}$ along (B.5) we have

$$
\dot{V}_{z}=2[\tilde{P}(t)-G]\left[-k_{i} V^{2} \tilde{P}(t)+k_{i} Y+k_{i} X\right]
$$

By applying Jensen's inequality (D.1), we obtain

$$
2 G^{2} \leq \int_{t-T}^{t}(\xi-t+T) \dot{\tilde{P}}^{2}(\xi) d \xi
$$

whereas via Jensen's inequalities (D.2) and (D.3) we find

$$
\begin{aligned}
X^{2} & =\frac{k_{p}^{2}}{k_{i}^{2} T^{4}}\left(\int_{t-T}^{t} \int_{\xi-T}^{\xi} v^{2}(\xi) \dot{\tilde{P}}(\theta) d \theta d \xi\right)^{2} \\
& \leq \frac{k_{p}^{2} v_{M}^{4}}{k_{i}^{2} T^{4}}\left(\int_{t-T}^{t} \int_{\xi-T}^{\xi} \dot{\tilde{P}}(\theta) d \theta d \xi\right)^{2} \\
& \leq \frac{k_{p}^{2} v_{M}^{4}}{k_{i}^{2} T^{2}} \int_{t-T}^{t} \int_{\xi-T}^{\xi} \dot{\tilde{P}}^{2}(\theta) d \theta d \xi \\
Y^{2} & =\frac{1}{T^{4}}\left(\int_{t-T}^{t} \int_{\xi-T}^{\xi} \int_{\theta}^{t} v^{2}(\xi) \dot{\tilde{P}}(\tau) d \tau d \theta d \xi\right)^{2} \\
& \leq \frac{v_{M}^{4}}{T^{4}}\left(\int_{t-T}^{t} \int_{\xi-T}^{\xi} \int_{\theta}^{t} \dot{\tilde{P}}(\tau) d \tau d \theta d \xi\right)^{2} \\
& \leq \frac{v_{M}^{4}}{T} \int_{t-T}^{t} \int_{\xi-T}^{\xi} \int_{\theta}^{t} \dot{\tilde{P}} 2(\tau) d \tau d \theta d \xi
\end{aligned}
$$

with $v_{M}$ given by (14). To compensate the $G$-term, similarly to (Fridman \& Shaikhet 2016), we will use

$$
V_{G}=\frac{k_{i}}{T} r \int_{t-T}^{t}(\xi-t+T)^{2} \dot{\tilde{P}}^{2}(\xi) d \xi, \quad r>0 .
$$

We have

$$
\dot{V}_{G}=k_{i} r T \dot{\tilde{P}}^{2}(t)-\frac{2 k_{i}}{T} r \int_{t-T}^{t}(\xi-t+T) \dot{\tilde{P}}^{2}(\xi) d \xi
$$

Then, due to (B.9)

$$
\dot{V}_{G} \leq k_{i} r T \dot{\tilde{P}}^{2}(t)-\frac{4 k_{i}}{T} r G^{2} .
$$

For the $X$-term, we consider

$$
V_{X}=\frac{k_{p}^{2}}{k_{i} T^{3}} r_{1} \int_{t-T}^{t} \int_{\xi-T}^{\xi}(\xi-t+T) \dot{\tilde{P}}^{2}(\theta) d \theta d \xi, \quad r_{1}>0 .
$$

Then,

$$
\dot{V}_{X}=\frac{k_{p}^{2}}{k_{i} T^{3}} r_{1}\left(T \int_{t-T}^{t} \dot{\tilde{P}}^{2}(\xi) d \xi-\int_{t-T}^{t} \int_{\xi-T}^{\xi} \dot{\tilde{P}}^{2}(\theta) d \theta d \xi\right)
$$

Via (B.10), we have

$$
\dot{V}_{X} \leq \frac{k_{p}^{2}}{k_{i} T^{2}} r_{1} \int_{t-T}^{t} \dot{\tilde{P}}^{2}(\xi) d \xi-\frac{k_{i}}{v_{M}^{4} T} r_{1} X^{2}
$$

To compensate the positive term in the right-hand side of (B.17), we employ

$$
\tilde{V}_{X}=\frac{k_{p}^{2}}{k_{i}} s \int_{t-T}^{t}(\xi-t+T) \dot{\tilde{P}}^{2}(\xi) d \xi, \quad s>0 .
$$

We have

$$
\dot{\tilde{V}}_{X}=\frac{k_{p}^{2}}{k_{i}} s\left(T \dot{\tilde{P}}^{2}(t)-\int_{t-T}^{t} \dot{\tilde{P}}^{2}(\xi) d \xi\right) .
$$

From (B.17) and (B.19), it follows that

$$
\begin{aligned}
\dot{V}_{X}+\dot{\tilde{V}}_{X} \leq & \frac{k_{p}^{2} T}{k_{i}} s \dot{\tilde{P}}^{2}(t)-\frac{k_{i}}{v_{M}^{4} T} r_{1} X^{2} \\
& -\frac{k_{p}^{2}}{k_{i}}\left(s-\frac{1}{T^{2}} r_{1}\right) \int_{t-T}^{t} \dot{\tilde{P}}^{2}(\xi) d \xi
\end{aligned}
$$


To compensate $Y$ in (B.8), we consider

$V_{Y}=\frac{k_{i}}{T^{2}} q \int_{t-T}^{t} \int_{\xi-T}^{\xi} \int_{\theta}^{t}(\xi-t+T) \dot{\tilde{P}}^{2}(\tau) d \tau d \theta d \xi, \quad q>0$.

Then,

$$
\begin{aligned}
\dot{V}_{Y}= & \frac{k_{i}}{T} q \int_{t-T}^{t} \int_{\xi-T}^{\xi} d \theta d \xi \cdot \dot{\tilde{P}}^{2}(t) \\
& +\frac{k_{i}}{T} q \int_{t-T}^{t} \int_{\theta}^{t} \dot{\tilde{P}}^{2}(\tau) d \tau d \theta \\
& -\frac{k_{i}}{T^{2}} q \int_{t-T}^{t} \int_{\xi-T}^{\xi} \int_{\theta}^{t} \dot{\tilde{P}}^{2}(\tau) d \tau d \theta d \xi \\
= & \frac{k_{i} T}{2} q \dot{\dot{P}^{2}}(t)+\frac{k_{i}}{T} q \int_{t-T}^{t} \int_{\theta}^{t} \dot{\tilde{P}}^{2}(\tau) d \tau d \theta \\
& -\frac{k_{i}}{T^{2}} q \int_{t-T}^{t} \int_{\xi-T}^{\xi} \int_{\theta}^{t} \dot{\tilde{P}}^{2}(\tau) d \tau d \theta d \xi .
\end{aligned}
$$

Further, by using (B.11) we have

$$
\dot{V}_{Y} \leq \frac{k_{i} T}{2} q \dot{\tilde{P}}^{2}(t)+\frac{k_{i}}{T} q \int_{t-T}^{t} \int_{\theta}^{t} \dot{\tilde{P}}^{2}(\tau) d \tau d \theta-\frac{k_{i}}{v_{M}^{4} T} q Y^{2}
$$

To cancel the double integral term in the right-hand side of (B.22), we additionally employ

$$
\tilde{V}_{Y}=\frac{k_{i}}{T} q \int_{t-T}^{t} \int_{\theta}^{t}(\theta-t+T) \dot{\tilde{P}}^{2}(\tau) d \tau d \theta
$$

Then,

$$
\begin{aligned}
\dot{\tilde{V}}_{Y}= & \frac{k_{i}}{T} q \int_{t-T}^{t}(\theta-t+T) d \theta \dot{\tilde{P}}^{2}(t) \\
& -\frac{k_{i}}{T} q \int_{t-T}^{t} \int_{\theta}^{t} \dot{\tilde{P}}^{2}(\tau) d \tau d \theta \\
= & \frac{k_{i} T}{2} q \dot{\tilde{P}}^{2}(t)-\frac{k_{i}}{T} q \int_{t-T}^{t} \int_{\theta}^{t} \dot{\tilde{P}}^{2}(\tau) d \tau d \theta .
\end{aligned}
$$

From (B.14), (B.20), (B.22) and (B.24), we obtain

$$
\begin{aligned}
& \dot{V}_{G}+\dot{V}_{X}+\dot{\tilde{V}}_{X}+\dot{V}_{Y}+\dot{\tilde{V}}_{Y} \\
& \leq k_{i} T\left(r+q+\frac{k_{p}^{2}}{k_{i}^{2}} s\right) \dot{\tilde{P}}^{2}(t)-\frac{4 k_{i}}{T} r G^{2}-\frac{k_{i}}{v_{M}^{4} T} r_{1} X^{2} \\
& \quad-\frac{k_{i}}{v_{M}^{4} T} q Y^{2}-\frac{k_{p}^{2}}{k_{i}}\left(s-\frac{1}{T^{2}} r_{1}\right) \int_{t-T}^{t} \dot{\tilde{P}}^{2}(\xi) d \xi
\end{aligned}
$$

Employing (B.2) and further using Young's inequality and Jensen's inequality (3.87) in (Fridman 2014) we have

$$
\begin{aligned}
\dot{\tilde{P}}^{2}(t) & \leq \frac{v_{M}^{4}}{T^{2}}\left[k_{p} \int_{t-T}^{t} \dot{\tilde{P}}(\xi) d \xi+k_{i} \int_{t-T}^{t} \tilde{P}(\xi) d \xi\right]^{2} \\
& \leq \frac{2 v_{M}^{4}}{T^{2}}\left[k_{p}^{2}\left(\int_{t-T}^{t} \dot{\tilde{P}}(\xi) d \xi\right)^{2}+k_{i}^{2}\left(\int_{t-T}^{t} \tilde{P}(\xi) d \xi\right)^{2}\right] \\
& \leq \frac{2 v_{M}^{4}}{T}\left[k_{p}^{2} \int_{t-T}^{t} \dot{\tilde{P}}^{2}(\xi) d \xi+k_{i}^{2} \int_{t-T}^{t} \tilde{P}^{2}(\xi) d \xi\right]
\end{aligned}
$$

with $v_{M}$ given by (14). Substitution of (B.26) into (B.25) leads to the term $2 k_{i}^{3} v_{M}^{4}\left(r+q+\frac{k_{p}^{2}}{k_{i}^{2}} s\right) \int_{t-T}^{t} \tilde{P}^{2}(\xi) d \xi$. To cancel the latter term, we additionally employ

$$
V_{v_{M}}=2 k_{i}^{3} v_{M}^{4}\left(r+q+\frac{k_{p}^{2}}{k_{i}^{2}} s\right) \int_{t-T}^{t}(\xi-t+T) \tilde{P}^{2}(\xi) d \xi
$$

that leads to

$$
\dot{V}_{v_{M}}=2 k_{i}^{3} v_{M}^{4}\left(r+q+\frac{k_{p}^{2}}{k_{i}^{2}} s\right)\left(T \tilde{P}^{2}(t)-\int_{t-T}^{t} \tilde{P}^{2}(\xi) d \xi\right) .
$$

Define a Lyapunov functional as

$$
\mathcal{V}:=V_{z}+V_{G}+V_{X}+\tilde{V}_{X}+V_{Y}+\tilde{V}_{Y}+V_{v_{M}},
$$

where $V_{z}, V_{G}, V_{X}, \tilde{V}_{X}, V_{Y}, \tilde{V}_{Y}$ and $V_{v_{M}}$ are given by (B.7), (B.12), (B.15), (B.18), (B.21), (B.23) and (B.27), respectively. Note that Jensen's inequality (3.87) in (Fridman 2014)

$$
\int_{t-T}^{t} \phi^{2}(\xi) d \xi \geq \frac{1}{T}\left[\int_{t-T}^{t} \phi(\xi) d \xi\right]^{2}
$$

with $\phi(s)=(\xi-t+T) \dot{\tilde{P}}(\xi)$ leads to $V_{G} \geq k_{i} r G^{2}$, whereas Jensen's inequality (D.1) leads to $\tilde{V}_{X} \geq \frac{2 k_{p}^{2}}{k_{i}} s G^{2}$. Hence

$$
\begin{aligned}
\mathcal{V} & \geq V_{z}+V_{G}+\tilde{V}_{X} \\
& \geq\left[\begin{array}{c}
\tilde{P}(t) \\
G
\end{array}\right]^{\top}\left[\begin{array}{cc}
1 & -1 \\
* 1+k_{i} r+\frac{2 k_{p}^{2}}{k_{i}} s
\end{array}\right]\left[\begin{array}{c}
\tilde{P}(t) \\
G
\end{array}\right] \geq c \tilde{P}^{2}(t)
\end{aligned}
$$

with $T$-independent $c>0$. Thus, $\mathcal{V}$ is positive definite. Taking into account (B.8), (B.25) and (B.28), via (12), we arrive at

$$
\begin{aligned}
\dot{\mathcal{V}} \leq & k_{i} \eta^{\top} \Xi\left(k_{p}, k_{i}\right) \eta+\frac{k_{p}^{2}}{k_{i}}\left[-s+2 v_{M}^{4}\left(k_{i}^{2}(r+q)\right.\right. \\
& \left.\left.\left.+k_{p}^{2} s\right)+\frac{1}{T^{2}} r_{1}\right)\right] \int_{t-T}^{t} \dot{\tilde{P}}^{2}(\xi) d \xi \\
\leq & k_{i} \eta^{\top} \Xi\left(k_{p}, k_{i}\right) \eta, \quad \eta:=\operatorname{col}\{\tilde{P}(t), G, Y, X\}
\end{aligned}
$$

where $\Xi(\cdot, \cdot)$ is defined by (13). Therefore, conditions (7) are satisfied.

Since $k_{p}$ and $k_{i}$ appear only in the positive terms of (12) and $\Xi_{11}(\cdot, \cdot)$, the feasibility of LMIs (12) and (13) with the maximum value of $k_{p}^{M}>0$ and $k_{i}^{M}>0$ implies their feasibility for all $-k_{p}^{M} \leq k_{p} \leq k_{p}^{M}$ and $0<k_{i} \leq k_{i}^{M}$.

(ii) For $k_{p}^{M}=0,(\mathrm{~B} .5)$ holds with $X=0$, whereas (B.26) is changed by

$$
\dot{\tilde{P}}^{2}(t) \leq \frac{k_{i}^{2} v_{M}^{4}}{T^{2}}\left[\int_{t-T}^{t} \tilde{P}(\xi) d \xi\right]^{2} \leq \frac{k_{i}^{2} v_{M}^{4}}{T} \int_{t-T}^{t} \tilde{P}^{2}(\xi) d \xi
$$

Then choosing $\mathcal{V}$ as in (B.29) with $V_{X}=\tilde{V}_{X}=0$ and with $V_{v_{M}}$ changed by $\frac{1}{2} V_{v_{M}}$, and using arguments of (i) we arrive at the result. 


\section{Proof of Corollary 1}

(i) Averaging of (B.2) over $\left[t-\frac{T}{2}, t\right]$ for $t \geq \frac{T}{2}$ leads to (B.5) with

$$
\begin{aligned}
G & =\frac{2}{T} \int_{t-\frac{T}{2}}^{t}\left(\xi-t+\frac{T}{2}\right) \dot{\tilde{P}}(\xi) d \xi, \\
Y & =\frac{2}{T^{2}} \int_{t-\frac{T}{2}}^{t} \int_{\xi-T}^{\xi} \int_{\theta}^{t} v^{2}(\xi) \dot{\tilde{P}}(\tau) d \tau d \theta d \xi, \\
X & =-\frac{2 k_{p}}{k_{i} T^{2}} \int_{t-\frac{T}{2}}^{t} \int_{\xi-T}^{\xi} v^{2}(\xi) \dot{\tilde{P}}(\theta) d \theta d \xi .
\end{aligned}
$$

Then, choosing Lyapunov functional

$$
\begin{aligned}
\mathcal{V}= & z^{2}(t)+\frac{2 k_{i}}{T} r \int_{t-\frac{T}{2}}^{t}\left(\xi-t+\frac{T}{2}\right)^{2} \dot{\tilde{P}}^{2}(\xi) d \xi \\
& +\frac{2 k_{p}^{2}}{k_{i} T^{3}} r_{1} \int_{t-\frac{T}{2}}^{t} \int_{\xi-T}^{\xi}\left(\xi-t+\frac{T}{2}\right) \dot{\tilde{P}}^{2}(\theta) d \theta d \xi \\
& +\frac{k_{p}^{2}}{k_{i}} s \int_{t-T}^{t}(\xi-t+T) \dot{\tilde{P}}^{2}(\xi) d \xi \\
& +\frac{8 k_{i}}{3 T^{2}} q \int_{t-\frac{T}{2}}^{t} \int_{\xi-T}^{\xi} \int_{\theta}^{t}\left(\xi-t+\frac{T}{2}\right) \dot{\tilde{P}}^{2}(\tau) d \tau d \theta d \xi \\
& +\frac{4 k_{i}}{3 T} q \int_{t-T}^{t} \int_{\theta}^{t}(\theta-t+T) \dot{\tilde{P}}^{2}(\tau) d \tau d \theta \\
& +2 k_{i}^{3} v_{M}^{4}\left(\frac{r}{2}+q+\frac{k_{p}^{2}}{k_{i}^{2}} s\right) \int_{t-T}^{t}(\xi-t+T) \tilde{P}^{2}(\xi) d \xi
\end{aligned}
$$

with $r>0, r_{1}>0, s>0$ and $q>0$, we arrive at

$$
\dot{\mathcal{V}} \leq k_{i} \eta^{\top} \hat{\Xi}\left(k_{p}, k_{i}\right) \eta, \quad \eta:=\operatorname{col}\{\tilde{P}(t), G, Y, X\},
$$

where $\hat{\Xi}(\cdot, \cdot)$ is given by $(17)$.

(ii) The proof of (ii) is similar to (ii) of Proposition 2.

\section{Jensen's inequalities}

\section{Lemma 1 Denote}

$$
\begin{aligned}
\mathcal{G} & :=\int_{a}^{b} f(\xi) x(\xi) d \xi, \quad \mathcal{X}:=\int_{t-T}^{t} \int_{\xi-T}^{\xi} x(\theta) d \theta d \xi \\
\mathcal{Y} & :=\int_{t-T}^{t} \int_{\xi-T}^{\xi} \int_{\theta}^{t} x(\tau) d \tau d \theta d \xi
\end{aligned}
$$

where $a \leq b, f:[a, b] \rightarrow \mathbb{R}, x(\tau) \in \mathbb{R}^{n}$ and the integration concerned is well defined. Then, for any $n \times n$ matrix $R>0$ the following Jensen's inequalities hold:

$$
\begin{aligned}
\mathcal{G}^{\top} R \mathcal{G} & \leq \int_{a}^{b}|f(\xi)| d \xi \int_{a}^{b}|f(\xi)| x^{\top}(\xi) R x(\xi) d \xi, \\
\mathcal{X}^{\top} R \mathcal{X} & \leq T^{2} \int_{t-T}^{t} \int_{\xi-T}^{\xi} x^{\top}(\theta) R x(\theta) d \theta d \xi
\end{aligned}
$$

and

$$
\mathcal{Y}^{\top} R \mathcal{Y} \leq T^{3} \int_{t-T}^{t} \int_{\xi-T}^{\xi} \int_{\theta}^{t} x^{\top}(\tau) R x(\tau) d \tau d \theta d \xi .
$$

PROOF. Inequality (D.1) was proved in (Solomon \& Fridman 2013). We will prove (D.2) and (D.3). By Schur complement, the following holds

$$
\left[\begin{array}{rr}
x^{\top}(\tau) R x(\tau) & x^{\top}(\tau) \\
* & R^{-1}
\end{array}\right] \geq 0 .
$$

Integration of (D.4), respectively, from $\xi-T$ to $\xi$ in $\theta$, from $t-T$ to $t$ in $\xi$, and from $\theta$ to $t$ in $\tau$, from $\xi-T$ to $\xi$ in $\theta$, from $t-T$ to $t$ in $\xi$, where

$$
\int_{t-T}^{t} \int_{\xi-T}^{\xi} d \theta d \xi=T^{2}, \quad \int_{t-T}^{t} \int_{\xi-T}^{\xi} \int_{\theta}^{t} d \tau d \theta d \xi=T^{3},
$$

and application of Schur complement leads to (D.2) and (D.3). 